\title{
Therapeutic strategies for synchronous and multiple liver metastases from colorectal cancer
}

\author{
Shinji Osada, Hisashi Imai, Yoshiyuki Sasaki, Yoshihiro Tanaka, Nobuhisa Matsuhashi, \\ Naoki Okumura, Michitaka Nagase, Kenichi Nonaka, Kazuhiro Yoshida \\ Surgical Oncology, Gifu University School of Medicine, Gifu, Japan
}

\begin{abstract}
Metastasis in the liver is one of the most critical factors in the prognosis of patients with colorectal cancer. The incidence of synchronous liver metastasis has been found to be approximately $20-25 \%$, but the optimal timing of surgical resection remains controversial. Neoadjuvant chemotherapy has also been found to be beneficial not only for initially unresectable but also resectable synchronous metastases and traditional surgical strategies of hepatic resection with past chemotherapeutic regimens have been used less and less over the past several years. This review will discuss treatments in association with the recently developed chemotherapeutic regimens.
\end{abstract}

\section{Introduction}

Colorectal cancer (CRC) is the third most common cancer worldwide after lung and breast cancers. It is estimated to account for over one million new cases per year, half of which are fatal. ${ }^{1}$ The natural course of CRC will develop metastasis to the liver up to $50 \%$ of

Correspondence: : Shinji Osada, Surgical Oncology, Gifu University School of Medicine, 1-1 Yanagido, Gifu 501-1194, Japan.

Tel. +81.58230 .6233 - Fax: +81.58230 .1074$

E-mail: sting@gifu-u.ac.jp

Key words: colorectal cancer, surgical indication, synchronous and multiple liver metastasis, chemotherapy, epithelial-to-mesenchymal transition.

Contributions: SO, HI, YS, study conception and design; YT, NO, MN, acquisition of data; KN, MN, analysis and interpretation of data; SO, drafting of manuscript and critical revision; KY, supervision.

Conflict of interests: the authors declare no potential conflict of interests.

Received for publication: 21 March 2012.

Revision received: 15 May 2012.

Accepted for publication: 28 May 2012.

This work is licensed under a Creative Commons Attribution NonCommercial 3.0 License (CC BY-NC 3.0).

CC Copyright S. Osada, et al., 2012

Licensee PAGEPress, Italy

Oncology Reviews 2012; 6:e9

doi:10.4081/oncol.2012.e9 patients. $^{2}$ The incidence of synchronous liver metastasis, commonly defined as liver metastasis occurring within 12 months of the primary colon cancer, has been detected in approximately $20-25 \%$ of cases. ${ }^{3}$ Expansion of multidisciplinary care with advances in surgical procedure and technique in the past decade has resulted in simultaneous resection being the standard treatment of choice because of its safety and efficiency. ${ }^{4}$ However, the optimal timing of surgical resection of synchronous metastasis remains controversial, and guidelines regarding the upper limits of operative indications for synchronous metastases have not yet been defined. In addition, neoadjuvant chemotherapy has also been found to be beneficial not only for initially unresectable but also resectable synchronous metastases. ${ }^{5,6}$ After the development of combinations of 5 -fluorouracil/folinic acid with irinotecan (FOLFIRI) or oxaliplatin (FOLFOX) treatment regimens, a prospective phase II study demonstrated that the response rate was $66 \%{ }^{7}$ and the maximum resection rate was $82 \% .{ }^{8}$ Traditional surgical strategies of hepatic resection in accordance with past chemotherapeutic regimens have been used less and less over the past several years. Therefore, this review will primarily discuss treatments in association with the FOLFOX/FOLFIRI chemotherapeutic regimens. Consideration of the timing of hepatectomy and whether it should be performed first or staged, should also take into account the contents and results of recent chemotherapeutic developments. ${ }^{9}$ This review will discuss chemotherapeutic strategies involving FOLFOX or FOLFIRI and/or hepatectomy.

\section{Timing of hepatectomy}

\section{Selection for simultaneous or staged hepatectomy}

Short- and long-term survival is reported to be worse in synchronous colon and liver metastasis. ${ }^{10}$ The surgical indications for resection of synchronous metastasis and the optimal timing of hepatectomy (simultaneous or staged) are still controversial and widely debated. ${ }^{11}$ A recent report showed colectomy with hepatectomy to have equivalent short-term outcomes if hepatectomy was minor, whereas major hepatectomy was associated with a doubling of the total severe morbidity rate $(36.1 \%$ vs $17.6 \%)$ and a nearly 6 -fold increase in mortality $(8.3 \%$ vs $1.4 \%$ ) in comparison with staged resection. ${ }^{12}$ Based on these results, a staged operation for synchronous and multiple hepatic nodules has been recommended with a delay of at least three months after the primary resection. In contrast, other studies showed that simultaneous resection results in similar mortality and morbidity rates but shorter hospital stays than staged operations, despite the fact that both groups have similar operative times, intraoperative blood loss and complications. ${ }^{9,13}$ Recent studies also showed simultaneous resection to enhance safety compared with staged operations and recommended that simultaneous resection be performed. ${ }^{4,14,15}$ According to the safe- 
ty concepts for such surgical procedures, simultaneous colorectal and liver resections have been evaluated as grade $\mathrm{C}$ under the recommended guidelines. ${ }^{16}$ However, no randomized trials have been reported.

Neoadjuvant chemotherapy before hepatectomy should be considered to reduce intrahepatic recurrence, even if the tumors are resectable. ${ }^{17}$ Pre- or postoperative FOLFOX chemotherapy versus surgery alone in patients with resectable liver metastases was evaluated in the final report from the European Organization for Research and Treatment of Cancer (EORTC) 40983 randomized trial. ${ }^{5}$ According to these results, chemotherapy was found to be significantly better than surgery alone at inducing 3-year progression-free survival (42.4\% vs $33.2 \%, \mathrm{P}=0.025)$. The study proposed the establishment of a new standard whereby pre-operative chemotherapy is to be performed even if the tumor is resectable. However, the use of oxaliplatin has caused concern because its use might worsen pre-operative liver function. ${ }^{18}$ Resection of both intra- and extra-hepatic metastases should be considered if all metastatic sites can be completely resected and the disease is controlled by chemotherapy. ${ }^{19} \mathrm{~A}$ recent report showed that neoadjuvant FOLFOXRI administered for 3-6 months is actually safe, ${ }^{20}$ and no consensus exists concerning operative mortality and morbidity rates. ${ }^{21,22}$ Even in the case of repeat hepatectomy, the operation itself was reported to be safe and to offer survival benefit. ${ }^{23}$ In cases of recurrence, $70 \%$ were observed within 12 months after the initial liver operation, with $92 \%$ observed within 24 months, ${ }^{24}$ and for disease isolated to the liver, repeat hepatic resection led to favorable patient survival. $^{25,26}$ The criteria for the selection of patients for hepatic re-resection included the ability to achieve an $\mathrm{R} 0$ resection, the disease-free interval, solitary recurrences and operative risk. The 5-year diseasefree survival rate after repeat hepatectomy was almost the same as that achieved after initial hepatectomy (26\% vs $25 \%){ }^{27}$

\section{Recently recommended strategy}

A reverse strategy has been proposed for patients with advanced synchronous colorectal cancer metastases, and in particular for patients in whom the primary tumor is located in the rectum. ${ }^{28}$ According to this report, pre-operative chemotherapy is followed by resection of the colorectal metastases and then by resection of the colorectal primary in a second operation. The risk for progression of metastases while the patient is undergoing treatment for the primary tumor is a cause for concern. In some patients, colorectal cancer metastases become unresectable during this interval because the delay until the resection of metastases is frequently longer than three months. ${ }^{29}$ The major limitation of this strategy is that extensive resections, including major or extended hepatectomy, are associated with increased mortality and severe morbidity rates (up to $8 \%$ and $36 \%$, respectively) when combined with resection of the primary tumors..$^{30} \mathrm{~A}$ recent study of the feasibility and safety of this reverse strategy demonstrated morbidity and mortality rates of $19 \%$ and $0 \%$, respectively, and a 3 -year overall survival rate of $83 \% .{ }^{28}$ Another study showed the reverse strategy to be associated with postoperative morbidity and mortality rates of $31 \%$ and $4 \%$, respectively, and a 3 -year survival rate of $79 \%{ }^{11}$ The new reverse approach includes the risk that during the period between chemotherapy and liver resection the primary tumor might become obstructive. This rare possibility can easily be solved by performing the Hartmann procedure; studies show that this liver first approach is a safe procedure that brings satisfactory results. ${ }^{31}$ The primary tumor can be left in place without the need for resection in patients with stage IV disease who received palliative systematic chemotherapy for advanced unresectable metastatic disease. ${ }^{32}$ In this report, among 233 patients with advanced stage IV colorectal cancer, only 26 patients (11\%) had symp- toms related to the primary tumor, which was similar to the $15 \%$ rate seen in the subset of patients with rectal primaries left in place. The reverse strategy might not be considered in patients with an initially circumferential obstructive tumor. However, in most patients, this strategy is appropriate because it establishes early control of stage IV disease both systemically and in the liver, and it is also associated with an effective response in the primary tumor. ${ }^{33}$ These concepts might be critical and it is expected that a series of clinical studies will be planned.

\section{Concepts emerging from basic studies}

Hepatocyte growth factor/scatter factor (HGF/SF) and its receptor, cMet, are well known to relate to liver regeneration. Its overexpression or activation has also been studied in the progression of $\mathrm{CRC} ;{ }^{34}$ therefore, the c-Met pathway is believed to play a critical role in the carcinogenesis of CRC. A report of clinical cases demonstrated that liver metastasis was significantly higher in the group with high expression of c-Met. However, in CRC cases with liver metastasis, despite high-grade immunodetection of c-Met activity in the primary tumor, these cases changed to low-grade activity in liver metastasis sites. An experimental mouse study also showed that expression of c-Met decreased from culture conditions to metastasis with time and tumor size dependency. ${ }^{34}$

Recently, some novel concepts for cancer growth and invasion have been derived from epithelial-to-mesenchymal transition (EMT), whereby a cancer cell changes its cellular phenotype from a local growing type and acquires an invasive and/or metastatic ability. ${ }^{35}$ EMT has been well recognized at the invasive margins of cancer masses, but not in localized tumors. After migrating to sites distant from the primary tumor, mesenchymal-epithelial transition (MET) is also associated with
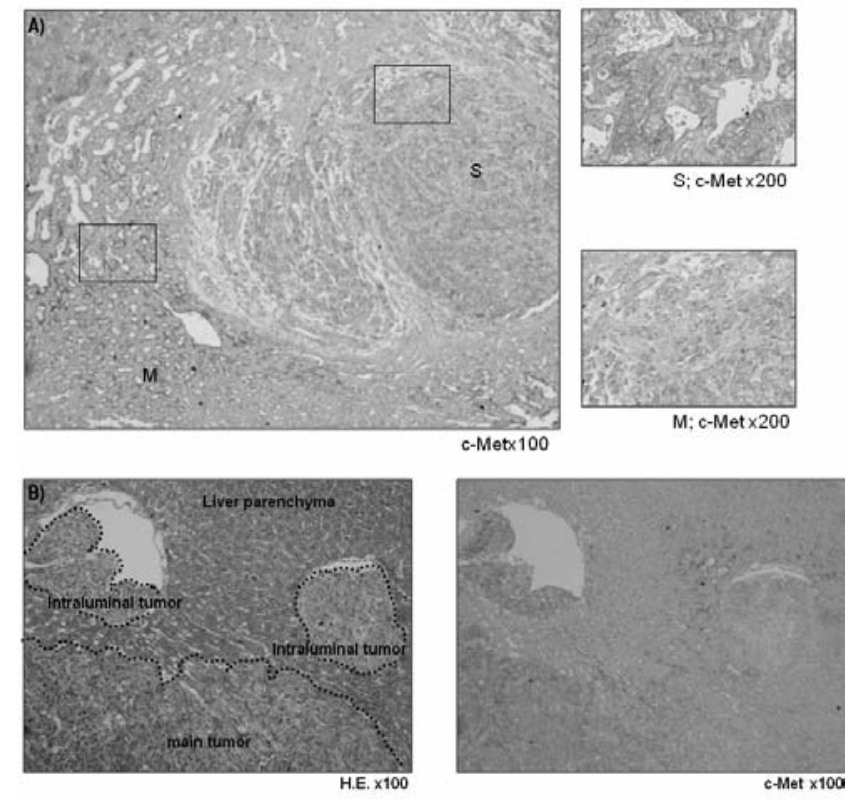

Figure 1. c-Met expression in liver metastatic sites. A) In the main tumor, c-Met expression was reduced in the central area but remained at high levels in satellite lesions. B) In the peripheral area of the main tumor, c-Met expression was diminished in the space completely occupied by vessels, but remained high in the space devoid of vessels. 
increases in mass-building activity. ${ }^{36} \mathrm{HGF}$ itself is well known as a SF, and is involved in the regulation of not only cell growth but also cell motility and morphology. ${ }^{38}$ Carcinoma cells with HGF/SF have already been demonstrated in relation to EMT, ${ }^{39}$ suggesting that primary CRC cells with highly expressed c-Met gain motility due to HGF/receptor activation for progression to the vessels and/or distant organs. Therefore, it seems that the HGF/c-Met system mediates cancer progression from local expansion to distant area metastasis via the process of EMT, and is down-regulated in mass formation at secondary sites via the process of MET.

Further studies using immunohistochemistry have added to these results (Figure 1). The expression of c-Met was clearly reduced in satellite lesions, despite expression remaining high in the central area of the same tumor. Even within single tumors, there was a difference in c-Met expression whereby it was increased in the growing invasive periphery but decreased in the established central regions. There may be a concern that as a treatment for metastastic liver tumors, hepatec- tomy could induce tumor growth in the residual liver. ${ }^{40}$ As serum levels of HGF increase following hepatectomy, the suggestion that this could prompt the growth of c-Met over-expressing colorectal cancer cells has been discussed.

\section{Clinical studies}

Some specific factors have been identified that give indications for the treatment policies required. ${ }^{9}$ Hepatectomy should be selected first if the resection can be performed safely and with a possibility of cure, with no limit on the size or number of tumors. However, where curative resection is not performed for reasons such as the presence of tumors in other organs, chemotherapy should be selected first, and the timing of possible radical hepatectomy immediately planned. ${ }^{9}$

The overall 5-year survival rate and median survival time (MST) for

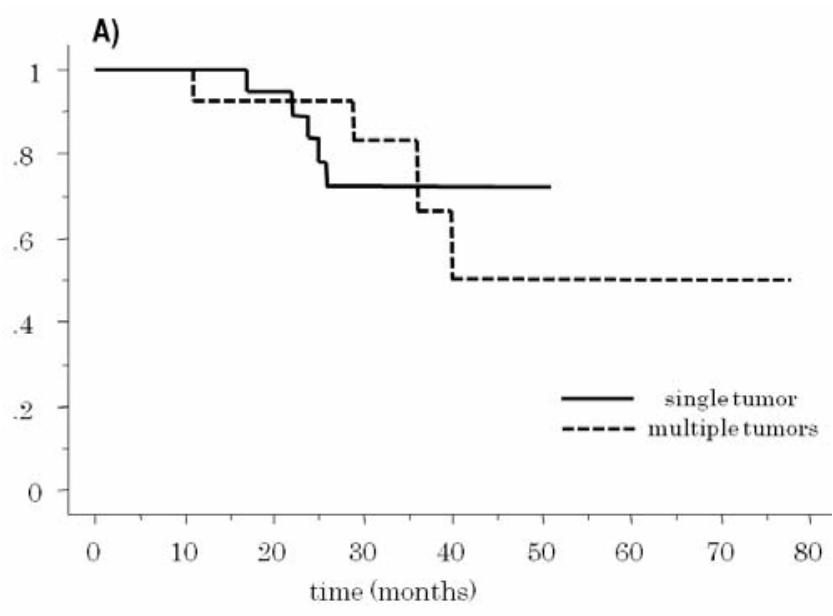

B)

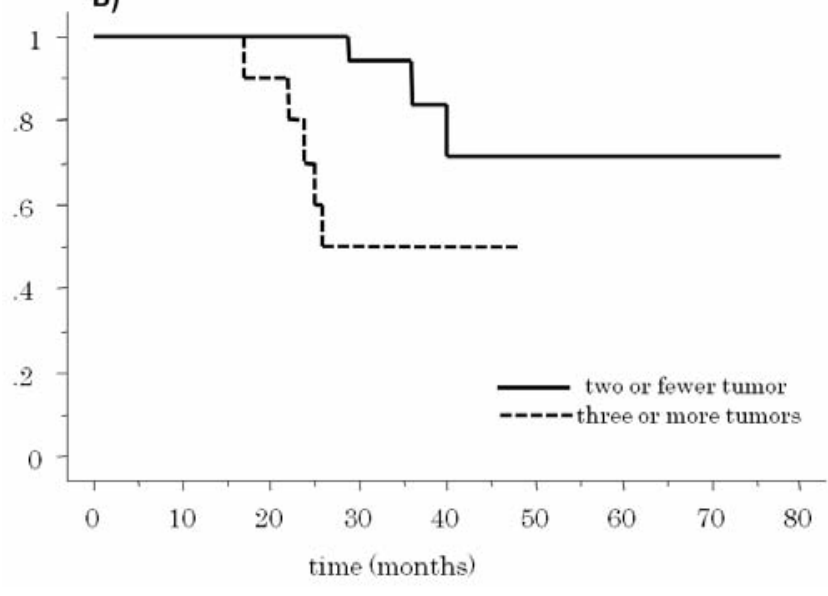

Figure 2. Clinical outcomes compared for tumor numbers. A) Among all patients studied, the 3-year survival rate and median survival time (MST) of patients with a single tumor (continuous line, $72 \%$ and $37.9 \pm 16.8$ months; $N=12$ ) were similar to those of patients with multiple tumors (dotted line, $65 \%$ and $28.2 \pm 13.1$ months; $\mathrm{N}=25)$. B) The 3-year survival rate and MST of patients with two or fewer tumors (continuous line, $83 \%$ and $36.6 \pm 14.0$ months; $\mathrm{N}=18)$ were significantly better $(\mathrm{P}=\mathbf{0 . 0 1 2 7})$ than those of patients with three or more tumors (dotted line, 65\% and 24.0 \pm 13.6 months; $\mathrm{N}=19$ ).

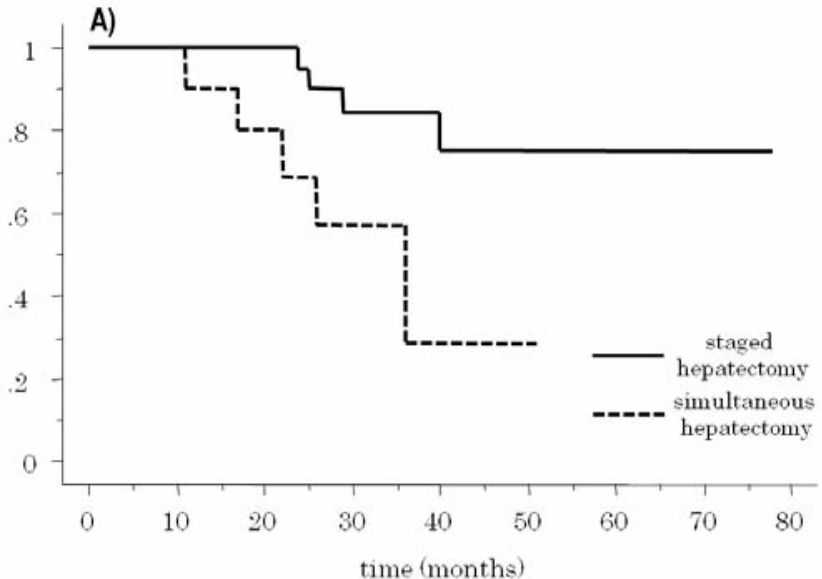

B)

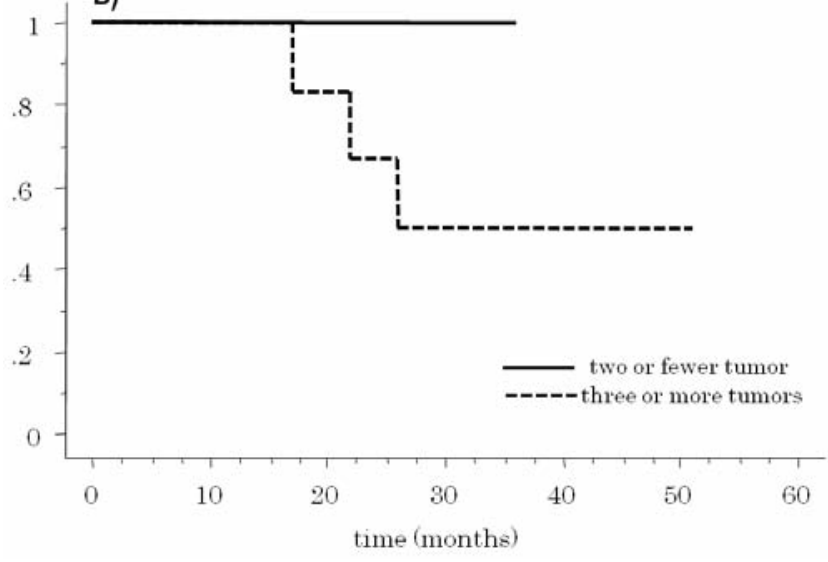

Figure 3. Clinical outcomes for simultaneous hepatectomy. A) For patients in whom synchronous liver tumors were detected, 3-year survival rate and median survival time (MST) after staged hepatectomy (continuous line, $82 \%$ and $34.5 \pm 14.9$ months: $\mathrm{N}=16$ ) were significantly better $(P=0.0467)$ than those after simultaneous hepatectomy (dotted line, $29 \%$ and $23.9 \pm 13.6$ months; $\mathrm{N}=10)$. B) Furthermore, despite the MST (29.7 \pm 8.5 months) after simultaneous hepatectomy for a single tumor being similar to that for multiple tumors $(23.4 \pm 15.7$ months $)$ for patients with two or fewer tumors (continuous line; $\mathrm{N}=6)$ the MST (34.8 \pm 11.0 months) was significantly longer than for patients with three or more tumors (dotted line, 17.0 \pm 9.9 months; $N=10$ ). 
patients in our recent study ${ }^{41}$ were $61.2 \%$ and $31.0 \pm 15.2$ months, respectively. Of these patients, the 3 -year survival rate (55\%) and MST $(28.4 \pm 15.4$ months) of patients in whom synchronous liver metastasis was detected were clearly poorer than those of patients with metachronous tumors ( $100 \%$ and $39.9 \pm 10.8$ months). Among all patients studied, the 3-year survival rate and MST in patients with a single tumor were similar to those of patients with multiple tumors. However, these indicators were significantly better $(\mathrm{P}=0.0127)$ for patients with two or fewer tumors than for patients with three or more tumors (Figure 2). Furthermore, in patients in whom synchronous liver tumors were detected, the 3-year survival rate and MST after staged hepatectomy were significantly better than those after simultaneous hepatectomy $(\mathrm{P}=0.0467)$, and the MST for patients with two or fewer tumors was significantly longer than that for patients with three or more tumors (Figure 3). The MST after simultaneous hepatectomy for a single tumor (29.7 \pm 8.5 months) was similar to that for multiple tumors (23.4 \pm 15.7 months). In contrast, after staged hepatectomy, the MSTs for patients with these factors were similar (single $v s$ multiple tumors, $40.7 \pm 18.3$ months vs $30.8 \pm 11.6$ months; two or fewer vs three or more tumors, $37.1 \pm 15.1$ months $v$ s $26.1 \pm 16.2$ months). According to another report, patients with one or two liver metastases had a similar prognosis, and those with three or more lesions had a significantly decreased 5 -year survival, from $28 \%$ to $13 \%(\mathrm{P}<0.01) .{ }^{42}$ In a recent review, ${ }^{43}$ a significantly worse rate of disease-free survival after curative resection for liver metastases was seen in patients with positive lymph node metastases, synchronous development timing, tumor-free interval of less than 12 months, presence of extrahepatic disease, and higher numbers of tumors. With regard to tumor number, the difference between two or fewer and three or more tumors was more critical for disease-free survival than that between one and two tumors $(\mathrm{P}=0.001$ vs 0.082 ) and there was no clear difference when the cut-off point for tumor number was set at four. Another recent study also found three independent factors that were predictive of disease recurrence: three or more metastases at diagnosis, initial unresectability, and simultaneous colorectal surgery with hepatectomy. ${ }^{44}$ Therefore, it appears that tumor number is important for patient survival, and a cut-off point set at two tumors may be reasonable for accepting primary hepatectomy.

\section{Conclusions}

Clinical features of CRC indicate that the overexpression of c-Met is closely associated with liver metastasis. In liver metastatic lesions, although a comparative reduction in c-Met expression correlates well with tumor growth, there is still a relatively high expression at invasive sites. Therefore, hepatectomy might be selected if cancer can no longer be detected by microscopic examination. According to clinical studies, neoadjuvant chemotherapy improves the prognosis for patients with synchronous liver metastases. Particularly in patients with one or two tumors, primary hepatectomy will induce a favorable outcome with a diminished likelihood of tumor in the remnant. In contrast, where there are three or more tumors, it is best to plan staged hepatectomy, even if it is technically possible to remove these tumors in one procedure. Treatment strategies for CRC patients with liver metastases should involve the consideration of appropriate combinations of chemotherapy and surgery.

\section{References}

1. Kamangar F, Dores GM, Anderson WF. Patterns of cancer incidence, mortality, and prevalence across five continents: defining priorities to reduce cancer disparities in different geographic regions of the world. J Clin Oncol 2006;24:2137-50.

2. Lochan R, White SA, Manas DM. Liver resection for colorectal liver metastasis. Surg Oncol 2007;16:33-45.

3. National Institute for Health and Clinical Excellence. Improving Outcomes in Colorectal Cancers. 2004 Jun 23. Available from: http://www.nice.org.uk/csgcc

4. Sakamoto Y, Fujita S, Akasu T, et al. Is surgical resection justified for stage IV colorectal cancer patients having bilobar hepatic metastasis? - an analysis of survival of 77 patients undergoing hepatectomy. J Surg Oncol 2010;102:784-8.

5. Nordlinger B, Sorbye H, Glimelius B, et al. Perioperative chemotherapy with FOLFOX4 and surgery versus surgery alone for resectable liver metastases from colorectal cancer (EORTC Intergroup trial 40983): a randomised controlled trial. Lancet 2008; 371:1007-16.

6. Adam R, Pascal G, Castaing D, et al. Tumor progression while on chemotherapy: a contraindication to liver resection for multiple colorectal metastases? Ann Surg 2004;240:1052-64.

7. Falcone A, Ricci S, Brunetti I, et al. Phase III trial of infusional fluorouracil, leucovorin, oxaliplatin and irinotecan (FOLFOXIRI) compared with infusional fluorouracil, leucovorin and irinotecan (FOLFIRI) as first-line treatment for metastatic colorectal cancer. J Clin Oncol 2007;25:1670-6.

8. Ychou M, Viret F, Kramar A, et al. A tritherapy with fluorouracil/leucovorin, irinotecan and oxaliplatin (FOLFILINOX): a phase II study in colorectal cancer patients with unresectable liver metastases. Cancer Chemother Pharmacol 2008;62:195-201.

9. Osada S, Imai H, Sasaki Y, et al. Strategy for synchronous and multiple liver metastasis. Hepatogastroenterology 2012;59:198-203.

10. Robertson DJ, Stukel TA, Gottlieb DJ, et al. Survival after hepatic resection of colorectal cancer metastasis: a national experience. Cancer 2009;115:752-9.

11. Brouquet A, Mortenson MM, Vauthey JN, et al. Surgical strategies for synchronous colorectal liver metastases in 156 consecutive patients: classic, combined or reverse strategy? J Am Coll Surg 2010;210:934-41.

12. Reddy SK, Pawlik TM, Zorzi D, et al. Simultaneous resection of colorectal cancer and synchronous liver metastases: a multi-institutional analysis. Ann Surg Oncol 2007;14:4481-91.

13. Capussotti L, Ferrero A, Vigano L, et al. Major resection synchronous with colorectal surgery. Ann Surg Oncol 2007;14:195-201.

14. Moug SJ, Smith D, Leen E, et al. Evidence for synchronous operative approach in the treatment of colorectal cancer with hepatic metastasis: A case matched study. Euro J Surg Oncol 2010;36:36570.

15. Chen J, Li Q, Wang C, et al. Simultaneous vs. staged resection for synchronous colorectal liver metastases: a meta-analysis. Int $\mathrm{J}$ Colorectal Dis 2011;26:191-9.

16. Hillingso JG, Wille-Jorgensen P. Staged or simultaneous resection of synchronous liver metastases from colorectal cancer: a systematic review. Colorectal Dis 2009;11:3-10.

17. Kanazawa A, Shiozawa M, Inagaki D, et al. Risk factors for intrahepatic recurrence after curative surgical treatment of colorectal liver metastases. Hepatogastroentrology 2010;57:1183-6.

18. Nakano H, Oussulzoglou E, Rosso E, et al. Sinusoidal injury increases mortality after major hepatectomy in patients with colorectal metastases receiving preoperative chemotherapy. Ann Surg 2008;247:118-24.

19. de Haas RJ, Wicherts DA, Adam R. Resection of colorectal liver metastases with extrahepatic diseases. Dig Surg 2008;25:461-6.

20. Msai G, Loupakis F, Pollina L, et al. Long-term outcome of initially unresectable metastatic colorectal cancer patients treated with 5fluorouraci//leucovorin, oxaliplatin, and irinotecan (FOLFOXIRI) 
followed by radical surgery of metastases. Ann Surg 2009;249:420-5.

21. Pawlik TM, Olino K, Gleisner AL, et al. Preoperative chemotherapy for colorectal liver metastases: impact on hepatic histology and postoperative outcome. J Gastrointest Surg 2007;11:863-8.

22. Zorzi D, Laurent A, Pawlik TM, et al. Chemotherapy-associated hepatotoxicity and surgery for colorectal liver metastases. Br J Surg 2007;97:274-86.

23. Adam R, Pascal G, Azoulay D, et al. Liver resection for colorectal metastases. The third hepatectomy. Ann Surg 2003;238:871-84.

24. Langenhoff BS, Krabbe PF, Ruers TJ. Efficacy of follow-up after surgical treatment of colorectal liver metastases. Eur J Surg Oncol 2009;35:180-6.

25. Tanaka K, Shimada H, Matsuo K, et al. Outcome after simultaneous colorectal and hepatic resection for colorectal cancer with synchronous metastases. Surgery 2004;136:650-9.

26. Antoniou A, Lovegrove RE, Tilney HS, et al. Meta analysis of clinical outcome after first and second liver resection for colorectal metastases. Surgery 2007;141:9-18.

27. Metcalfe MS, Mullin EJ, Maddern GJ. Choice of surveillance after hepatectomy for colorectal metastases. Arch Surg 2004;139:749-54.

28. Mentha G, Majno PE, Andres A, et al. Neoadjuvant chemotherapy and resection of advanced synchronous liver metastases before treatment of the colorectal primary. Br J Surg 2006;93:872-8.

29. Bosset JF, Collette L, Calais G, et al. Chemotherapy with preoperative radiotherapy in rectal cancer. N Eng J Med 2006;355:1114-23.

30. Reddy SK, Pawlik TM, Zorzi D, et al. Simultaneous resections of colorectal cancer and synchronous liver metastases: a multi-institutional analysis. Ann Surg Oncol 2007;14:3481-91.

31. Mentha G, Roth AD, Terraz S, et al. "Liver first" approach in the treatment of colorectal cancer with synchronous liver metastases. Dig Surg 2008;25:430-5.

32. Brouquet A, Mortenson MM, Vauthey JN, et al. Surgical strategies for synchronous colorectal liver metastases in 156 consecutive patients: classic, combined or reverse strategy? Am Col Surg 2010;210:934-41.

33. Poultsides GA, Servais EL, Saltz LB, et al. Outcome of primary tumor in patients with synchronous stage IV colorectal cancer receiving combination chemotherapy without surgery as initial treatment. J Clin Oncol 2009;27:3379-84.

34. Matsui S, Osada S, Tomita H, et al. Clinical significance of aggressive hepatectomy for colorectal liver metastasis, evaluated from the HGF/c-Met pathway. Int J Oncol 2010;37:289-97.

35. Lee JM, Dedhar S, Kalluri R, Thompson EW. The epithelial-mesenchymal transition: new insights in signalling, development, and disease. J Cell Biol 2006;172:973-81.

36. Vincan E, Barker N. The upstream components of the Wnt signalling pathway in the dynamic EMT and MET associated with colorectal cancer progression. Clin Exp Metastasis 2008;25:657-63.

37. Rubin C, Gur G, Yarden Y. Negative regulation of receptor tyrosine kinases: unexpected links to $\mathrm{c}$-Cbl and receptor ubiquitylation. Cell Res 2005;15:66-71.

38. Osada S, Kanematsu M, Imai H, et al. Evaluation of extracellular signal regulated kinase expression and its relation to treatment of hepatocellular carcinoma. J Am Coll Surg 2005;201:405-11.

39. Zhang A, Wang MH, Dong Z, Yang T. Prostaglandin E2 is a potent inhibitor of epithelial-to-mesenchymal transition: interaction with hepatocyte growth factor. Am J Physiol Renal Physiol 2006;291: F1323-31.

40. Harun N, Nikfarjam M, Muralidharan V, Christophi C. Liver regeneration stimulates tumor metastases. J Surg Res 2007;138:284-90.

41. Osada S, Imai H, Sasaki Y, et al. Surgical indications for multiple and synchronous and liver metastases from colorectal cancer. Hepatogastroenterology [In press].

42. Taylor M, Forster J, Langer B, et al. A study of prognostic factor for hepatic resection for colorectal metastases. Am J Surg 1997;173: 467-71.

43. Settmacher U, Dittmar Y, Knosel T, et al. Predictors of long-term survival in patients with colorectal liver metastases: a single center study and review of the literature. Int J Colorectal Dis 2011;26: $967-81$.

44. de Haas RJ, Adam R, Wicherts DA, et al. Comparison of simultaneous or delayed liver surgery for limited synchronous colorectal metastases. Br J Surg 2010;97:1279-89. 